\title{
Mixed Boundary Conditions for Two-Dimensional Transient Heat Transfer Conduction under Lattice Boltzmann Simulations
}

\author{
R. Chaabane ${ }^{\dagger}$, F. Askri and S.B. Nasralla \\ Laboratoire d'Etudes des Systèmes Thermiques et Energétiques \\ Ecole Nationale des Ingénieurs de Monastir \\ Av. Ibn ElJazzar 5019 Monastir- Tunisie \\ $\dagger$ Corresponding Author Email: Raoudha.Chaabane@issatgb.rnu.tn
}

(Received April 25, 2010; accepted March 13, 2011)

\begin{abstract}
In this paper, lattice Boltzmann implementations of several types of boundary conditions are introduced and numerically demonstrated. A thermal lattice BGK model is used to simulate thermal fields for flows. The unknown thermal distribution functions at the boundary are subjected to the bounce back concept which is determined consistently with Dirichlet and/or Neumann and/or convective boundary conditions. A consistency analysis using heat transfer conduction is given and the algorithms are numerically tested in two space dimensions with respect to accuracy, numbers of iterations and CPU time. The method is used to simulate conduction transfer problems; numerical results and reference's solutions are found in satisfactory agreement for thermal fields.
\end{abstract}

Keywords: Lattice Boltzmann method, Boundary conditions, Dirichlet boundary, Newmann boundary, Convective boundary, 2D heat transfer.

\section{NOMENCLATURE}

$\begin{array}{cl}e_{i} & \begin{array}{l}\text { propagation speed in the direction } i \text { in the lattice, } \\ \mathrm{m} / \mathrm{s}\end{array} \\ \overrightarrow{e_{i}} & \begin{array}{l}\text { propagation velocity in the direction } i \text { in the } \\ \text { lattice, } \mathrm{m} / \mathrm{s}\end{array} \\ f_{i} & \begin{array}{l}\text { particle distribution function in the } i \text { direction, } K \\ T\end{array} \\ f_{i}^{(0)} & \begin{array}{l}\text { Dimensional temperature, } K \\ \text { equilibrium particle distribution function in the } i \text { - } \\ \text { direction, } \mathrm{K}\end{array} \\ t & \text { time, } \mathrm{s} \\ c_{p} & \text { Specific heat } m^{2} S^{-1} K^{-1} \\ \tau & \text { relaxation time in the } \mathrm{LBM}, \mathrm{s} \\ c_{s} & \text { Lattice sound velocity, } m s^{-1} \\ k & \text { Thermal conductivity, } W m^{-1} K^{-1} \\ \xi & \text { dimensionless time } \\ \theta & \text { Non dimensional temperature }\end{array}$

\section{INTRODUCTION}

Over the last decade the lattice-Boltzmann (LB) methods (Frisch et al. 1987; McNamara and Zanetti 1988; Higuera and Jiménez 1989; Succi et al. 1991; Benzi et al. 1992; Wolf-Gladrow 2000) have achieved great success as alternative and efficient numerical schemes in the simulation of a variety of transport phenomena in porous media (Chen and Doolen 1998; Spaid and Phelan 1997; Maier et al. 1998; Manz et al. $x, y \quad$ rectangular coordinates, $\mathrm{m}$

E, W, N, S east, west, north, south

$\begin{array}{cl}\vec{q}_{R} & \text { heat flux, } \mathrm{W} / \mathrm{m}^{2} \\ s & \text { position, } m \\ \omega_{i} & \text { weight factor corresponding to the } \\ & \text { direction } i \text { in a lattice } \\ \sigma & \text { Stefan-Boltzman constant } \\ \Delta t & \text { Time step, } s \\ \Delta x & \text { Lattice constant, } m \\ \Omega_{i} & \text { collision operator } \\ \rho & \text { density, } \mathrm{Kgm}^{-3} \\ \alpha & \text { Thermal diffusivity, } \mathrm{m}^{2} \mathrm{~S}^{-1} \\ * & \text { Dimensional variable }\end{array}$

2004; Maier et al. 1999; Bernsdorf et al. 2000; Clague et al. 2000; Hill et al. 2001; Békri et al. 2001; Drazer and Koplik 2001; Maier et al. 2002; Mishra et al. 2009; Kandhai et al. 2002; Schure et al. 2002; Kang et al. 2002; Zeiser et al. 2002; Békri et al. 2003), heat exchangers, cooling of electronic components, solar collectors, thermal insulation, air heating systems for solar dryers, passive solar heating and storage technology to name just a few (Ho et al. 2002; He et al. 1998; Xi et al. 1999; Takada et al. 2000; Wolf-Gladrow 
2000; Succi 2001; Nourgaliev et al. 2003; Zhu et al. 2005; Ho et al. 2002; Jiaung et al. 2001; Chatterjee and Chakraborty 2005). Besides their flexibility and accuracy in dealing with the confining geometry and actual boundary conditions, LB methods are inherently parallel and, thus, they are ideally suited for highperformance parallel computing. In contrast to the more conventional numerical schemes based on a discretization of macroscopic continuum equations, the LB method utilizes micro and meso-scale theoretical based on kinetic equations to recover the macroscopic Navier-Stokes equation for fluid motion in the longtime, large-scale limit Chen and Doolen (1998). The used algorithms have been simplified by the single relaxation time scheme of (Bhatnagar et al. 1954; Chen et al. 1991). Only a few reports have been published so far in which LB simulations also consider different benchmarks of Boundary conditions as Dirichlet or/and Newman Boundary conditions.

In this paper, an extension of the LB method was proposed based on convective Boundary conditions imposed at the cavity's walls. The resulting twodimensional problem was further restrained by assuming Dirichlet or/and Newman Boundary conditions. While this work addresses cases with the implementation of the lattice Boltzmann method (LBM) for the solution of conduction problems with Dirichlet or/and heat flux temperature or/and convective temperature boundary conditions.

The numerical approach that we present in this work can cope with any geometry and, thus, it will be particularly efficient in resolving details of the flow field that govern transport in a transient conduction or/and radiation equation.

The objective of the present work is to compare the performance of the present LBM code in solving transient conduction heat transfer in two-dimensional geometry with LBM code available in Mishra et al. (2009). For this, we consider two-dimensional rectangular geometry where one or two boundaries can be at prescribed heat flux conditions. The energy equation is solved using the LBM and obtained results are compared with reference's ones Mishra et al. (2009).

Then, we aims to extend the application of the LBM to solve heat conduction problems dealing with temperature as well as heat flux or/and convective boundary conditions.

To that end, we consider two-dimensional rectangular geometry where one boundary is at prescribed heat flux conditions and the remaining ones are subjected to a convective boundary condition.

A second benchmark problem dealing with transient conduction heat transfer in a two dimensional rectangular geometry where the four boundaries are subjected to a convective boundary condition is simulated.

Then, mixed boundary conditions is used showing the flexibility of the method and its efficiency to deal with any combination of these boundary conditions in order to model almost any 2D heat transfer situation subjected to varying boundary conditions.

\section{Thermal Lattice Boltzmann Model}

The heat transfer in a 2 -D rectangular enclosure is considered. Thermo-physical properties of the medium are assumed constant.

A variety of boundary conditions are imposed on the rectangular geometry.

For the problem under consideration, and in the absence of convection and radiation, the energy equation is given by

$\frac{\partial T}{\partial t}=\alpha \nabla^{2} T+Q$

Conventional numerical approaches such as the finite difference and finite element methods are based on the discretization of partial differential equations. In contrast, the LBM is based on the discrete Boltzmann kinetic equation. For heat transport problems, the internal energy evolution equation of the twodimensional nine-speed (D2Q9) lattice Boltzmann model is given by Qian et al. (1992)

$\frac{\partial f_{i}(\vec{r}, t)}{\partial t}+\overrightarrow{e_{i}} \nabla f_{i}(\vec{r}, t)=\Omega_{i}, \quad i=1,2,3, \ldots \ldots, b$

The collision operator $\Omega_{i}$ represents the rate of change of $f_{i}$ due to collisions. It incorporates all the physics and modelling of any particular problem at hand. The simplest model for $\Omega_{i}$ is the Bhatnagar-Gross-Krook (BGK) model (Drazer and Koplik 2001)

$\Omega_{i}=-\frac{1}{\tau}\left[f_{i}(\vec{r}, t)-f_{i}^{(0)}(\vec{r}, t)\right]$

$f_{i}$ is the particle distribution function denoting the number of particles at the lattice node $\vec{r}$ at time $t$ moving in direction $i$ with velocity $\overrightarrow{e_{i}}$ along the lattice link $\Delta \vec{r}=\overrightarrow{e_{i}} \Delta t$ connecting the nearest neighbours. $b$ is the number of directions in a lattice through which the information propagates.

The basis of the discrete velocity model is a finite set of virtual velocities $\overrightarrow{e_{i}}$ or equivalently, of virtual fluxes of the considered scalar field $T(\vec{r}, t)$ which given by

$T(\vec{r}, t)=\sum_{i=0}^{b} f_{i}(\vec{r}, t)$

The observed flux is expressed by

$\sum_{i=0}^{b} f_{i}(\vec{r}, t) \vec{e}_{i}$

The well-known D2Q9 lattice model (Fig.1) will be considered here. In that model, the set of $\overrightarrow{e_{i}}$ 's is such that they connect the point, on which the lattice stencil is centred, to its nearest neighbours on a spatial grid with uniform spacing in both coordinate directions. Any LBM advances the probability densities $f_{i}(\vec{r}, t)$ in time 
and thereby computes the evolution of the considered scalar. In the absence of external sources or fluxes for the scalar, the corresponding discrete evolution equation can be written in the following general form:

$$
\frac{\partial f_{i}(\vec{r}, t)}{\partial t}+\overrightarrow{e_{i}} \nabla f_{i}(\vec{r}, t)=-\frac{1}{\tau}\left[f_{i}(\vec{r}, t)-f_{i}^{(0)}(\vec{r}, t)\right]
$$
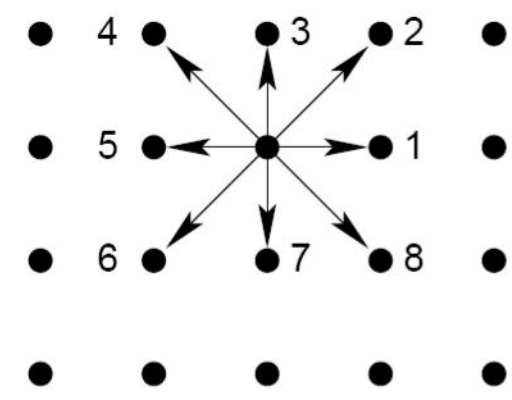

Fig.1. The D2Q9 lattice Boltzmann model.

It is a single-relaxation-time model with relaxation constant $\tau$ that can be related, via Chapmann-Enskog analysis, to the diffusivity of the medium. $f_{i}^{(0)}$ is the equilibrium distribution function.

The relaxation time can be related with the thermal diffusivity, the lattice velocity $C$ and the time step (Maier et al. 2002) by the following relation

$\tau=\frac{3 \alpha}{|C|^{2}}+\frac{\Delta t}{2}$

For the D2Q9 model in particular, the 9 velocities $\overrightarrow{e_{i}}$ and their corresponding weights $w_{i}$ are the following

$\vec{e}_{0}=(0,0)$

$\vec{e}_{i}=\left(\cos \left(\phi_{i}\right), \sin \left(\phi_{i}\right)\right) \cdot C \quad$ for $\quad \phi_{i}=(i-1) \pi / 2$ $i=1,2,3,4$

$\vec{e}_{i}=\sqrt{2}\left(\cos \left(\phi_{i}\right), \sin \left(\phi_{i}\right)\right) \cdot C \quad$ for $\phi_{i}=(i-5) \pi / 2+\pi$ $i=5,6,7,8$

$w_{i}=\frac{4}{9}$

$w_{i}=\frac{1}{9} \quad$ for $i=1,2,3,4$

$w_{i}=\frac{1}{36}$ for $i=5,6,7,8$

Where

$C=\Delta x / \Delta t=\Delta y / \Delta t, \Delta x$ and $\Delta t$ are the lattice space and the lattice time step size, respectively, which are set to unity. the weights satisfy the relation $\sum_{i=0}^{b} w_{i}=1$.

After discretization, and considering heat generation, Eq. (6) can be written as

$$
\begin{aligned}
f_{i}\left(\vec{r}+\overrightarrow{e_{i}} \Delta t, t+\Delta t\right) & =f_{i}(\vec{r}, t)-\frac{\Delta t}{\tau}\left[f_{i}(\vec{r}, t)-f_{i}^{(0)}(\vec{r}, t)\right] \\
& +w_{i} \Delta t Q^{*}
\end{aligned}
$$

Where $Q^{*}$ is the non dimensional heat generation and $w_{i}$ is the weight in corresponding direction.

To process Eq. (8), an equilibrium distribution function is required. For heat conduction problems, this is given by

$f_{i}^{(0)}(\vec{r}, t)=w_{i} T(\vec{r}, t)$

For the different sets of boundary condition, the boundaries are based on the properties of the known and unknown populations on each side as shown on Fig. 2.

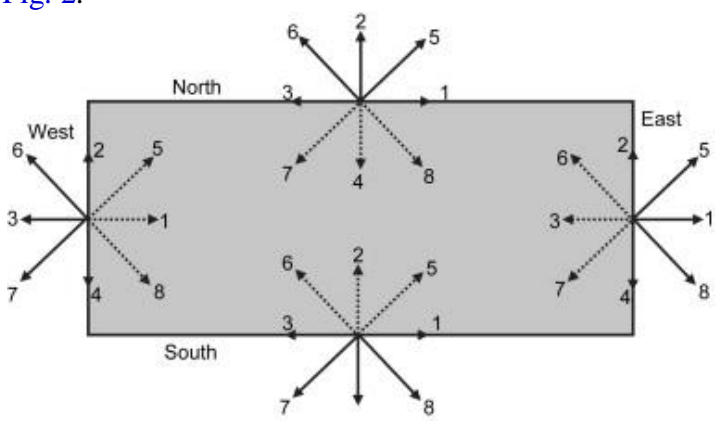

Fig. 2. Domain boundary conditions with known and unknown populations.

\section{RESUlTS AND DISCUSSION}

We consider transient heat conduction problems in 2-D Cartesian geometry with a variety of initial and boundary conditions.

Case 1: the four boundaries are at known temperatures The initial and the boundary conditions for case 1 are the following

Initial condition $\quad T(x, y, 0)=T_{\text {ref }}$

Boundary conditions

$T(x, 0, t)=0.25 T_{r \mathrm{e} f}$

$T(x, Y, t)=T(0, y, t)=T(X, y, t)=T_{\text {ref }}$

Steady state conditions were assumed to have been achieved when the temperature difference between two consecutive time levels at each lattice centre did not exceed $10^{-6}$. Non dimensional time was defined as $\xi=\alpha t / L^{2}$ where $\mathrm{L}$ is the characteristic length. $\Delta \xi$ was taken as $10^{-4}$.

To check the accuracy of the present LBM algorithm, the same problem was solved using the finite volume method and the results given by the two algorithms are compared with those available in the literature.

In Fig.3, the non dimensional centreline ( $\mathrm{x} / \mathrm{X}=0.5)$ temperature has been compared at different instants $\xi$ for the case 1 . 


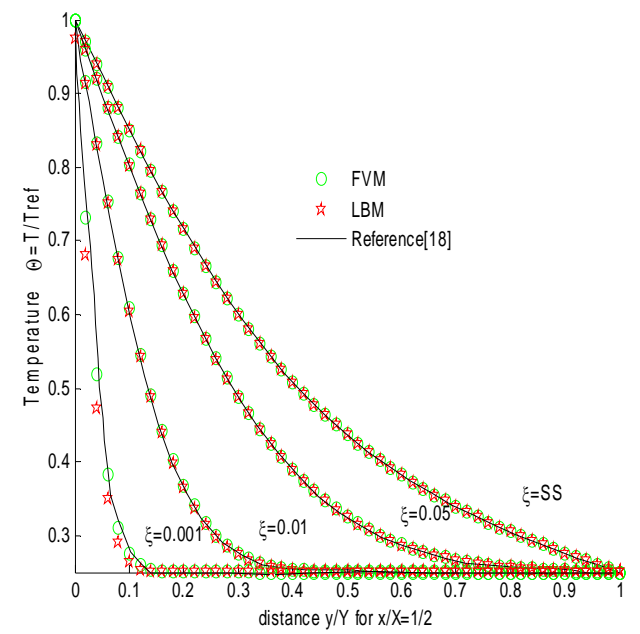

Fig. 3. Centerline $(\mathrm{x} / \mathrm{X}=0.5)$ temperature evolution for different instants (case 1).

Case 2: Effects of heat generation and the four boundaries are at specified temperatures.

In Fig. 4, the effects of volumetric heat generation are shown. The non dimensional volumetric heat generation is taken as unity.

Effect of heat generation is very less in the beginning compared to steady state because it takes some time to influence the temperature profile.

For the 2-D geometry, the number of iterations for a $50 \times 50$ grid is 3719 (135.95 seconds) compared to that cited at the literature 3257 (Mishra et al. 2009).

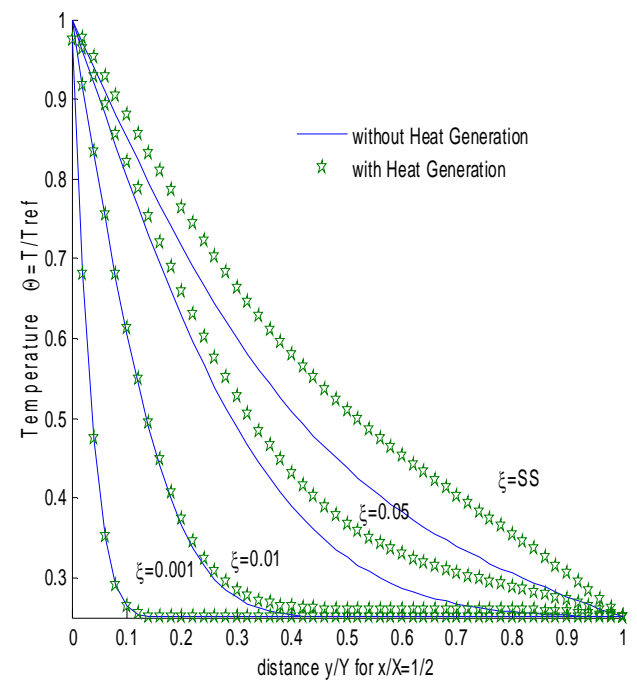

Fig. 4. Comparison of centreline $(x / X=0.5)$ temperature in the presence and the absence of heat generation.

The time-space evolution of the isotherms is plotted in Fig. 5 when the four boundaries are at specified temperatures (Dirichlet boundary condition) in the presence of non dimensional heat generation $\left(Q^{*}=2\right)$.
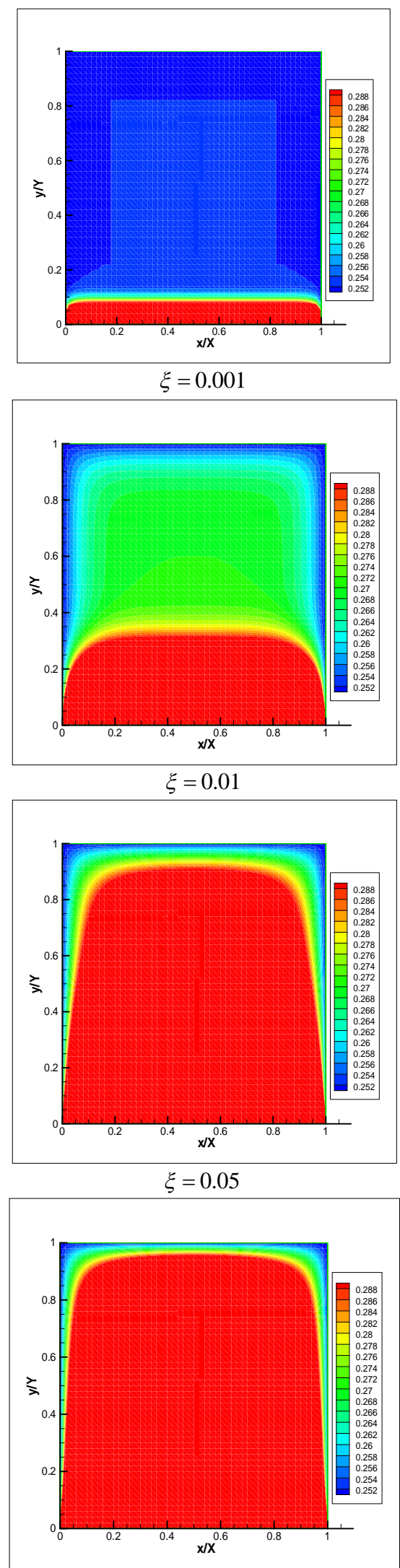

$\xi$ at steady state

Fig. 5. Isotherms when the four boundaries are at specified temperatures in the presence of non dimensional heat generation $\left(Q^{*}=2\right)$ for different $\xi$. 
Case3: The bottom and top boundaries are at prescribed fluxes and remaining two boundaries at known temperatures.

The system is initially at temperature $T_{E}$. For time $t>0$, the south and the north boundaries are subjected to heat fluxes $q_{T, S}$ and $q_{T, N}$, respectively. The east and the west boundaries are kept at temperatures $T_{E}$ and $T_{W}$, respectively.

Initial condition

$T(x, y, 0)=T_{0}$

Boundary conditions

$$
\begin{aligned}
& q(x, 0, t)=q_{s} \\
& q(x, Y, t)=q_{N} \\
& T(0, y, t)=T(X, y, t)=T_{0}
\end{aligned}
$$

It is seen from the Fig. 6 that the steady state results match exactly which each other.

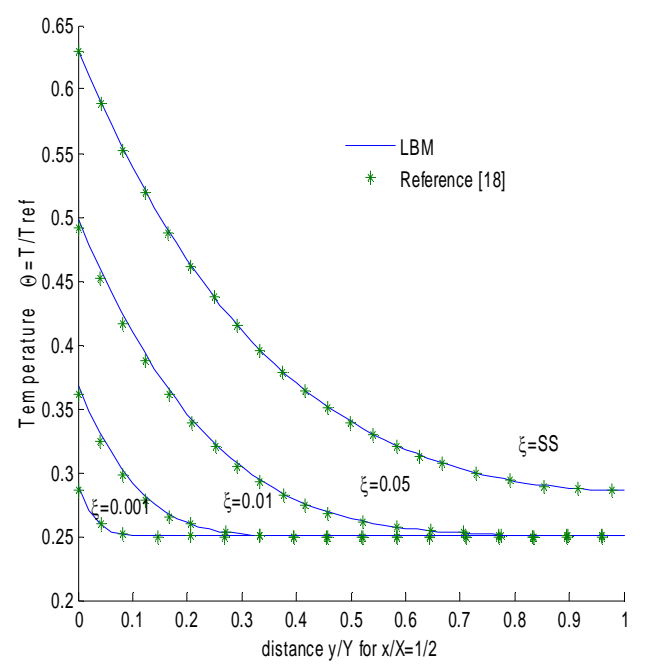

Fig. 6. Centreline $(\mathrm{x} / \mathrm{X}=0.5)$ temperature evolution for different instants (case3).

In Fig. 7, we present the time-space evolution of the isotherms when the bottom and the top boundaries are at prescribed fluxes and remaining two boundaries at known temperatures

Table 1CPU times (second) and number of iterations of the LBM code (case3)

\begin{tabular}{|c|c|c|c|}
\hline $\begin{array}{c}\text { Size } \\
\text { Lattices }\end{array}$ & iterations & $\begin{array}{c}\text { CPU time } \\
\text { (seconds) }\end{array}$ & $\begin{array}{c}\text { Temperature at } \\
\text { steady } \\
\text { state }(\mathrm{x} / \mathrm{X}=0.5)\end{array}$ \\
\hline $8 \times 8$ & 6251 & 12.42 & 0.44170 \\
\hline $12 \times 12$ & 6076 & 24.33 & 0.37722 \\
\hline $20 \times 20$ & 6051 & 53.026 & 0.34413 \\
\hline $50 \times 50$ & 6199 & 286.011 & 0.34493 \\
\hline
\end{tabular}

To have an idea of the number of iterations for the converged solutions and the CPU time, tests were performed with different lattices.
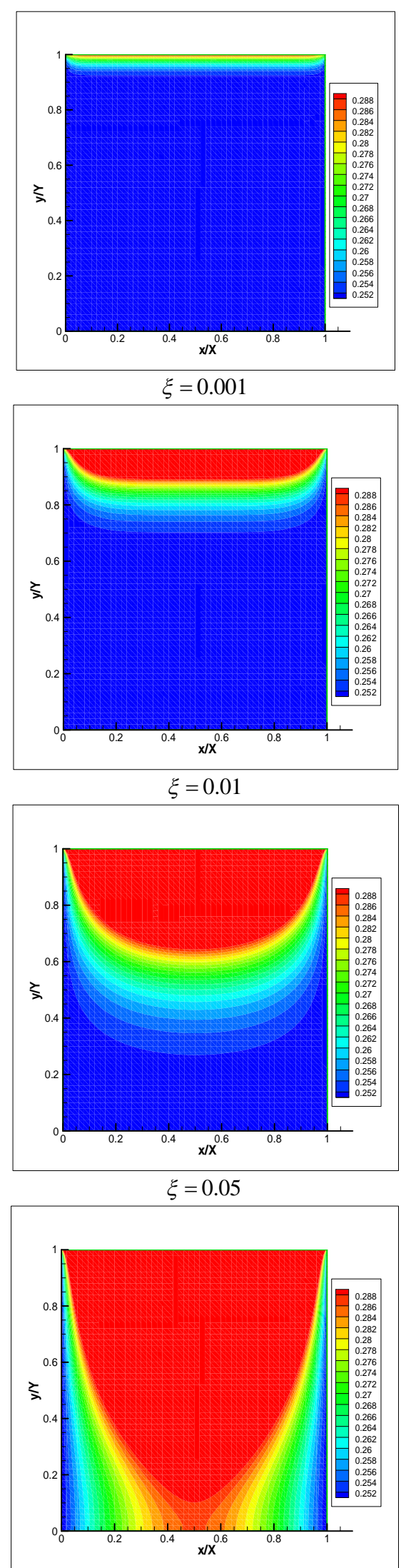

$\xi$ at steady state

Fig. 7. Isotherms when the bottom and the top boundaries are at prescribed fluxes and remaining two boundaries at known temperatures for different $\xi$. 
The LBM code was found to take slightly less number of iterations for the little lattices (Table 1). The effect of heat generation on CPU times (second) and number of iterations when all boundaries at known temperatures, was highlighted in Table 2.

Table 2 Effect of heat generation on CPU times (second) and number of iterations of the LBM code (case3)

\begin{tabular}{|c|c|c|}
\hline Lattice size & iterations & CPU time \\
\hline \multicolumn{3}{|c|}{ In the absence of heat generation } \\
\hline $50 \times 50$ & 6199 & 549.69 \\
\hline \multicolumn{3}{|c|}{ In the presence of heat generation } \\
\hline $50 \times 50$ & 6317 & 555.13 \\
\hline
\end{tabular}

\section{Case4:}

For the physical problem addressed in this section, the thermal boundary condition at the three side faces, are subjected to convective heat transfer boundary condition:

$$
-k \frac{\partial T}{\partial n}=q+h\left(T-T_{\infty}\right)
$$

where $h$ is the convective heat transfer coefficient. $n$ is the direction of outward normal to the surface concerned.

The bottom face is subjected to a heat flux, the temperature boundary condition is:

$\frac{\partial T}{\partial y}=q$

The corresponding non-dimensional forms of the boundary conditions obtained from Eqs. (21-22) are formulated as (shown in Fig. 8):

$$
\begin{aligned}
& -\frac{\partial \theta}{\partial n^{*}}=B i \theta \\
& \frac{\partial \theta}{\partial y^{*}}=0
\end{aligned}
$$

where $B i=h * l / k$ is the Biot number.

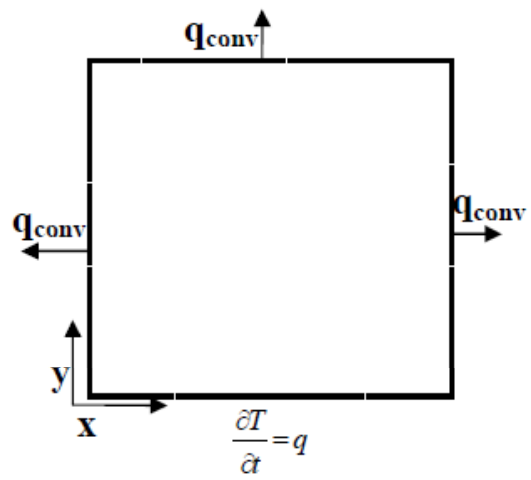

Fig. 8. Physical problem with mixed Newmann and convective boundary conditions.

In Fig. 9, we present the time-space evolution of the isotherms for case 4 .

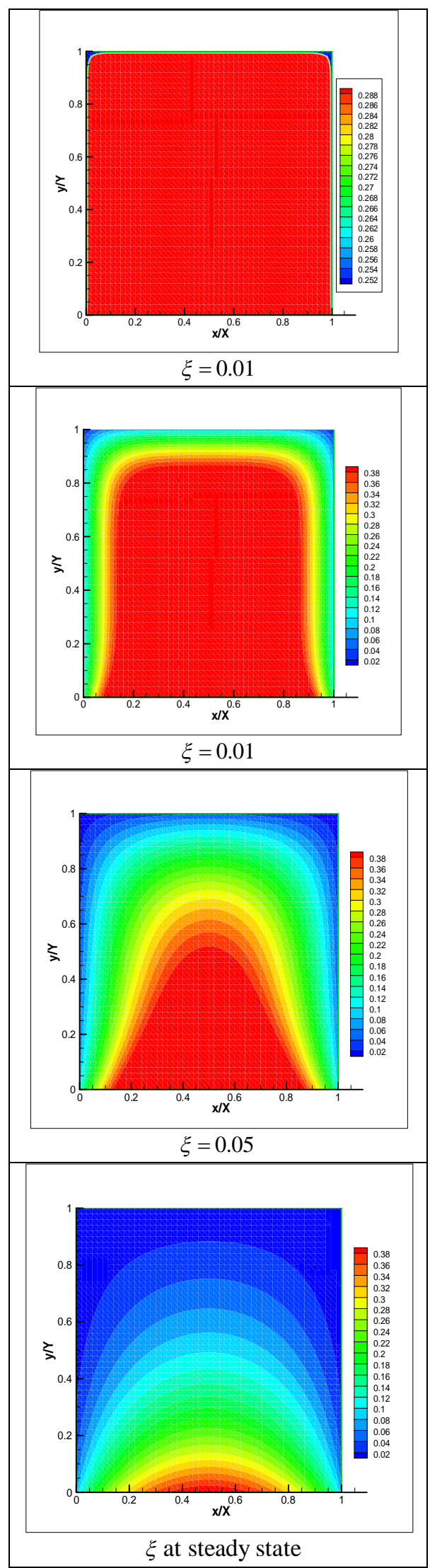

Fig. 9. isotherms when the bottom boundary is at prescribed flux and remaining three boundaries are convective ( $\mathrm{Qs}=1), \mathrm{Bi}=30$. 


\section{Case5:}

A four convective boundary conditions benchmark is studied in this section as presented in Fig. 10. In addition the medium is subjected to a heat generation conditions $\left(Q^{*}=1\right)$.

In order to analyze the effect of the non-dimensional Biot number, steady state non dimensional temperature is plotted for the case of four connectives boundaries as shown in Figs. 11, 12, and 13.

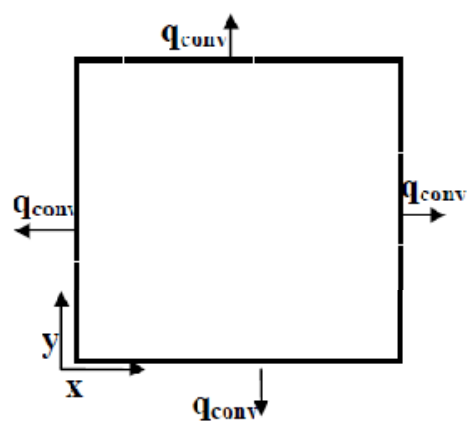

Fig. 10. Physical problem with convective boundary

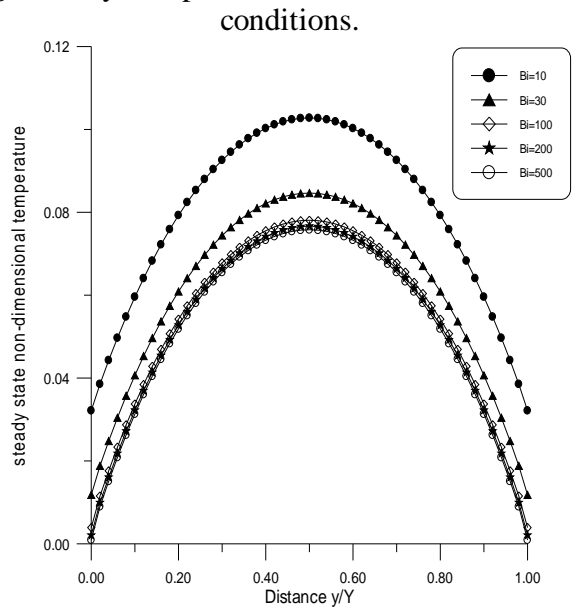

Fig. 11. Effect of Biot number on steady state non dimensional temperature, when the four boundaries are subjected to a convective boundary condition along $\mathrm{y} / \mathrm{Y}$ axis.

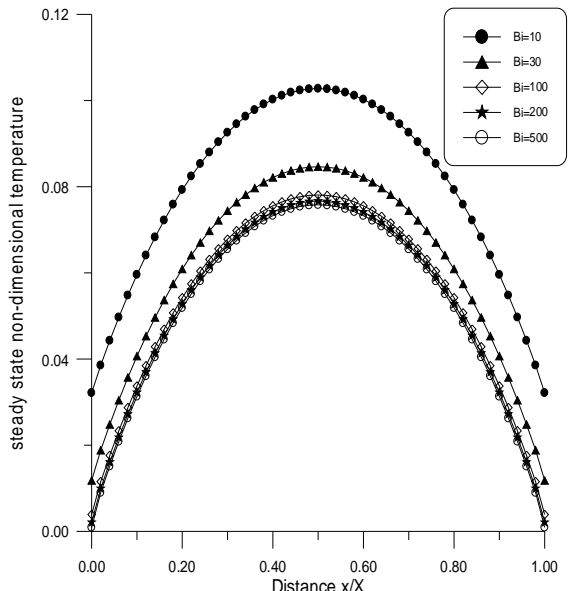

Fig. 12. Effect of Biot number on steady state nondimensional temperature, when the four boundaries are subjected to a convective boundary condition along $\mathrm{x} / \mathrm{X}$ axis.

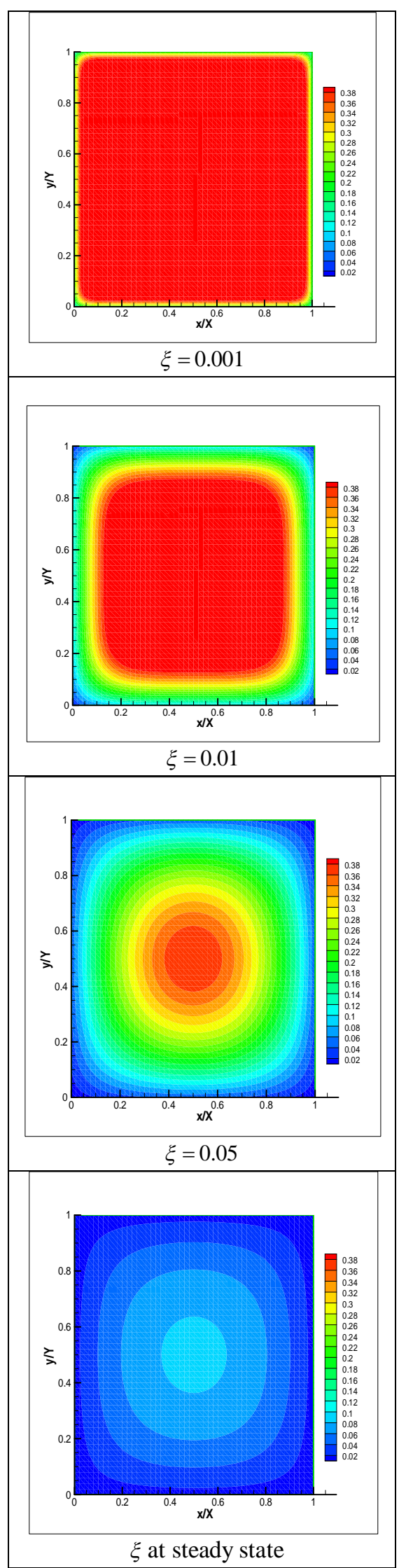

Fig. 13. Isotherms when the four boundaries are convective and the medium is subjected to a unity heat generation. 


\section{Case 6:}

The mixed convection/conduction/insulated boundary conditions example is constrained as shown in Fig.14. The distributions of isotherms are plotted in the Fig. 15 for different $\xi$. A unity dimensional Biot number is considered. $T_{0}=T_{W}=1$ and $T_{N}=5 T_{0}$

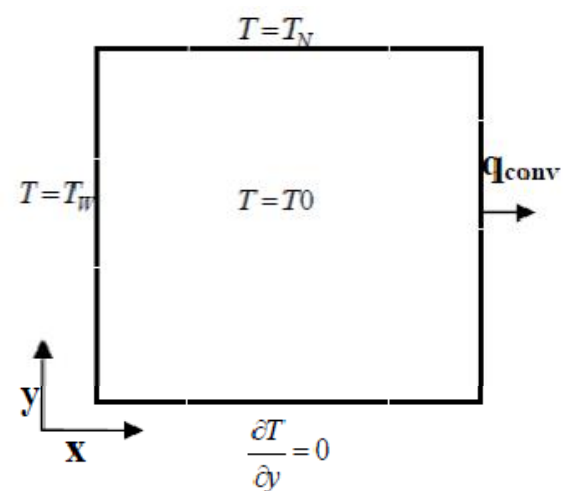

Fig. 14. Physical problem with mixed dirichlet/Newmann and convective boundary conditions.

The CPU times and the number of iterations of the LBM code ( $B i=30$ ) for a $50 \times 50$ grid is presented in the Table 3 for different convective boundary cases.

Table 3 CPU times (second) and number of iterations of the LBM code $(B i=30)$ for a $50 \times 50$ grid

\begin{tabular}{|c|c|c|c|}
\hline & iteration & $\begin{array}{c}\text { CPU } \\
\text { time } \\
(\mathrm{s})\end{array}$ & $\begin{array}{c}\text { Temperature at } \\
\text { steady state } \\
(\mathrm{x} / \mathrm{X}=\mathrm{y} / \mathrm{Y} 0.5)\end{array}$ \\
\hline $\begin{array}{c}4 \text { convective } \\
\text { boundaries }\end{array}$ & 5126 & 119.5 & 0.0845364 \\
\hline $\begin{array}{c}3 \text { convective } \\
\text { boundaries } \\
\text { Qs=0 }\end{array}$ & 7087 & 148.2 & 0.11306 \\
\hline $\begin{array}{c}3 \text { convective } \\
\text { boundaries } \\
\text { Qs=1 }\end{array}$ & 4381 & 104.9 & 0.216927 \\
\hline $\begin{array}{c}2 \text { convective } \\
\text { boundaries } \\
\text { Qs=1, Qn=0 }\end{array}$ & 5260 & 118.8 & 0.258066 \\
\hline
\end{tabular}

\section{Conclusions}

The lattice Boltzmann method is used to solve transient heat conduction problems in two dimensional geometries with uniform lattices.

Different sets of mixed boundary conditions were considered namely constant temperature and/or flux boundary conditions and/or convective boundary conditions.

The case of volumetric heat source in the medium was also taken up. In this paper, the problem was analyzed using two numerical approaches, lattice Boltzmann method and the finite volume method.

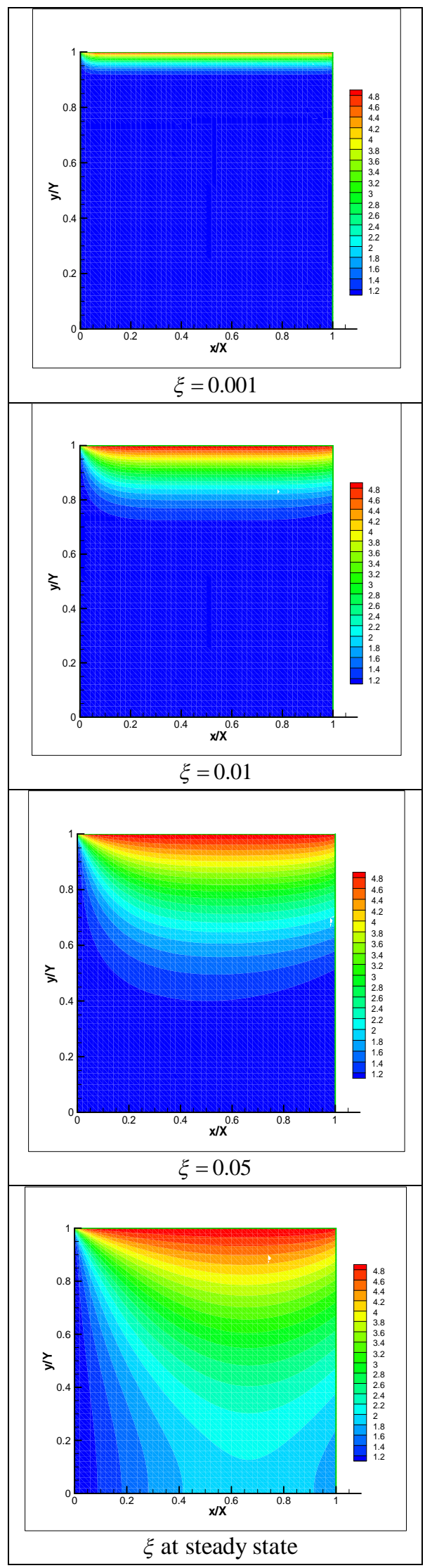

Fig. 15. Isotherms when the left the top boundaries are at specified temperatures, the bottom boundary is convective and the south one is insulated. 
Results given by the two numerical approaches are compared with those available in the literature and good agreement is obtained. On the other hand, the effect of lattice size is highlighted via the number of iterations and the CPU time. The considered $2 \mathrm{D}$ geometry is a simple one, to allow simple validation. Advection and radiation are omitted. Thus, it remains to demonstrate the viability of the LBM as heat diffusion-advection solver knowing that using LBM will allow us to output the temperature distribution in an extremely simple and accurate way.

\section{REFERENCES}

Békri, S., O. Vizika, J.F. Thovert and P.M. Adler (2001). Binary two-phase flow with phase change in porous media. International Journal of Multiphase Flow 27, 477-526.

Békri, S., J. Howard, J. Muller and P.M. Adler (2003). Electrical resistivity index in multiphase flow through porous media. Transport in Porous Media $51,41-65$.

Benzi, R., S. Succi and M. Vergassola (1992). The lattice Boltzmann equation-theory and applications. Physics Reports 222, 145-197.

Bernsdorf, J., G. Brenner and F. Durst (2000). Numerical analysis of the pressure drop in porous media flow with lattice Boltzmann (BGK) automata. Computer Physics Communications 129, 247-255.

Bhatnagar, P., E. Gross and M. Krook (1954). A model for collision processes in gases: I. small amplitude processes in charged and neutral one-component systems. Physical Review 94, 511-525.

Chatterjee, D. and S. Chakraborty (2005). An enthalpybased Lattice Boltzmann model for diffusion dominated solid-liquid phase transformation. Physics Letters 341, 320-330.

Chen, S. and G.D. Doolen (1998). Lattice Boltzmann method for fluid flows. Annual Review of Fluid Mechanics 30, 329-364.

Chen, S., H. Chen, D. Martinez and W.H. Matthaeus (1991). Lattice Boltzmann model for simulation of magnetohydrodynamics. Physical Review Letters 67, 3776-3779.

Clague, D.S., B.D. Kandhai, R. Zhang, P.M.A. Sloot (2000), Hydraulic permeability of (un)bounded fibrous media using the lattice Boltzmann method. Physical Review E 61, 616-625.

Drazer, G. and J. Koplik (2001). Tracer dispersion in two-dimensional rough fractures. Physical Review E 63, Art. No. 056104.

Frisch, U., D. d'Humieres, B. Hasslacher, Lallemand, Y. Pomeau and J.P. Rivet (1987). Lattice gas hydrodynamics in two and three dimensions. Complex Systems 1, 649-707.
He, X., S. Chen and G.D. Doolen (1998). A novel hermal model for the Lattice Boltzmann method in incompressible limit. Journal of Computational Physics 146, 282-300.

He, X. and L. Luo (1997). Theory of the lattice Boltzmann method: from the Boltzmann equation to the lattice Boltzmann equation. Phys. Rev. E 56, 6811.

Higuera, F.J. and J. Jiménez (1989). Boltzmann approach to lattice gas simulations. Europhysics Letters 9, $663-668$.

Hill, R.J., D.L. Koch and A.J.C. Ladd (2001). Moderate-Reynolds-number flows in ordered and random arrays of spheres. Journal of Fluid Mechanics 448, 243-278.

Ho, J.R., C.P. Kuo, W.S. Jiaung and C.J. Twu (2002). Lattice Boltzmann scheme for hyperbolic heat conduction equation. Numerical Heat Transfer Part B 41, 591-607.

Jiaung, W.S., J.R. Ho and C.P. Kuo (2001). Lattice Boltzmann Method for heat conduction problem with phase change. Numerical Heat Transfer, Part B 39, 167-187.

Kandhai, D., D. Hlushkou, A.G. Hoekstra, P.M.A. Sloot, H. Van As and U. Tallarek (2002). Influence of stagnant zones on transient and asymptotic dispersion in macroscopically homogeneous porous media. Physical Review Letters 88, Art. No. 234501.

Kang, Q.H., D.X. Zhang and S.Y. Chen (2002). Unified lattice Boltzmann method for flow in multiscale porous media. Physical Review E 66, Art. No. 056307.

Maier, R.S., D.M. Kroll, R.S. Bernard, S.E. Howington, J.F. Peters and H.T. Davis (2002). Enhanced dispersion in cylindrical packed beds. Philosophical Transactions of the Royal Society of London A 360, 497-506.

Maier, R.S., D.M. Kroll, H.T. Davis, R.S. Bernard (1999). Simulation of flow in bidisperse sphere packings. Journal of Colloid and Interface Science 217, 341-347.

Maier, R.S., D.M. Kroll, Y.E. Kutsovsky, H.T. Davis and R.S. Bernard (1998). Simulation of flow through bead packs using the lattice Boltzmann method. Physics of Fluids 10, 60-74.

Manz, B., L.F. Gladden and P.B. Warren (2004). Flow and dispersion in porous media: lattice-Boltzmann and NMR studies. AIChE Journal 45, 1845-1854. Copyright (2004) John Wiley \& Sons, Ltd. Numerical simulation of electroosmotic flow. Int. J. Numer. Meth. Fluids 46, 507-532. 
R. Chaabane et al. / JAFM, Vol. 4, No. 2, Special Issue, pp. 89-98, 2011.

McNamara, G. and G. Zanetti (1988). Use of the Boltzmann equation to simulate lattice-gas automata. Physical Review Letters 61, 2332-2335.

Mishra, S.C., M. Bittagopal, K. Tanuj, B.S.R. Krishna (2009). Solving transient heat conduction problems on uniform and non uniform lattices using the Lattice Boltzmann Method. International Communications in Heat and Mass Transfer 36, 322-328.

Nourgaliev, R.R., T.N. Dinh, T.G. Theofanous and D. Joseph (2003). The Lattice Boltzmann equation method: theoretical interpretation, numerics and implications. International Journal of Multiphase Flow 29, 117-169.

Qian, Y.H., D. d'Humieres and P. Lallemand (1992). Lattice BGK models for the Navier-Stokes equation. Europhysics Letters 17, 479-484.

Schure, M.R., R.S. Maier, D.M. Kroll and H.T. Davis (2002). Simulation of packed-bed chromatography utilizing high-resolution flow fields: comparison with models. Analytical Chemistry 74, 6006-6016.

Spaid, M.A.A. and F.R. Jr. Phelan (1997). Lattice Boltzmann methods for modeling microscale flow in fibrous porous media. Physics of Fluids 9, 24682474.

Succi, S. (2001). The Lattice Boltzmann Method for Fluid Dynamics and Beyond. Oxford University Press: New York.

Succi, S., R. Benzi and F. Higuera (1991). The latticeBoltzmann equation-a new tool for computational fluid dynamics. Physica D 47, 219230 .

Takada, N., M. Misawa, A. Tomiyama and S. Fujiwara (2000). Numerical simulation of two- and threedimensional two-phase fluid motion by Lattice Boltzmann method. Computer Physics Communications 129, 233-236.

Wolf-Gladrow, D.A. (2000). Lattice Gas Cellular Automata and Lattice Boltzmann Models: An Introduction. Springer Verlag, Berlin-Heidelberg.

Xi, H., G. Peng and S.H. Chou (1999). Finite-volume Lattice Boltzmann schemes in two and three dimensions. Physical Review E 60, 3380-3388.

Yoshino, M. and T. Inamuro (2003). Lattice Boltzmann simulations for flow and heat mass transfer problems in a three dimensional porous structure. International Journal for Numerical Methods in Fluids 43, $183-198$

Zeiser, T., M. Steven, H. Freund, P. Lammers, G. Brenner, F. Durst and J. Bernsdorf (2002). Analysis of the flow field and pressure drop in fixed-bed reactors with the help of lattice Boltzmann simulations. Philosophical
Transactions of the Royal Society of London A $360,507-520$.

Zhu, L., D. Tretheway, L. Petzold and C. Meinhart (2005). Simulation of fluid slip at 3D hydrophobic micro channel walls by the Lattice Boltzmann method. Journal of Computational Physics 202, 181-195. 